\section{Adaptation to uniocular image magnification: Is the underlying shift proprioceptive?*}

\author{
WILLIAM EPSTEIN† \\ University of Wisconsin, Madison, Wisconsin 53706
}

Ss wore a $4 \%$ overall size lens (OSL) before one eye while they engaged in $20 \mathrm{~min}$ of binocular exploration of a typical indoor environment. Tests before and after the exposure period were conducted to determine whether or not OSL exposure leads to shifts in depth judgments based on binocular disparity. No significant shifts were observed. These results were applied to an evaluation of two contrasting hypotheses that have been proposed to explain adaptation to meridional size lens (MSL).

Recent experiments (Epstein, 1971; Epstein \& Morgan, 1970; Mack \& Chitayat, 1970) have provided evidence of adaptation to optically produced transformations of binocular disparity. Epstein transformed the relationship between objective depth and binocular disparity by introducing a meridional size lens (MSL) in front of one of S's eyes. The MSL, whose axis of orientation was vertical (90 deg), magnified the retinal image in the horizontal meridian and altered the transverse binocular disparities that are normally associated with points separated in depth. Following $20 \mathrm{~min}$ of MSL exposure to an extralaboratory indoor environment, judgments of depth, secured in a modified Howard-Dolman apparatus, exhibited significant adaptive shifts.

Epstein attributed the shift to an acquired recalibration or reevaluation of binocular disparity. In other words, it was contended that the fundamental change underlying the adaptive shift was a modification of the relationship between binocular retinal input and the perceptual correlate, i.e., between disparity and perceived depth. Mack and Chitayat (1970) also advocated this view of the adaptive shifts observed in their experiment, which involved binocular exposure to opposite rotations of the retinal images of the two eyes.

One of the principal unsettled issues in the study of adaptation concerns the locus of adaptation (Epstein, 1967, Chap. 9; Rock, 1966). Ámong the competing views, the hypothesis of proprioceptive shift (Harris, 1965) has been prominent. According to this hypothesis, the shift underlying adaptation involves the felt position of parts of the body. When changes in registered eye position are included in the domain of proprioceptive shift, the hypothesis gains considerable

\footnotetext{
*This research was supported by NIH Grant MH 16390-03.

†Address: Psychology Building, University of Wisconsin, Charter at West Johnson Streets, Madison, Wisconsin 53706.
}

generality (e.g., Pick, Hay, \& Martin, 1969). The hypothesis has been applied recently to the case of MSL adaptation: "It would be consistent with [the proprioceptive shift hypothesis ] to account for the change in apparent depth with the assumption that S's apparent vantage point was one of asymmetrical convergence. The temporal course of adaptation would be characterized by an increase in the registered magnitude of asymmetrical convergence, that is, $\mathrm{S}$ would come to sense his convergence direction to be more and more asymmetrical as exposure time increased. Thus, with $\mathbf{S}$ actually looking straight ahead and the aniseikonic lens over the right eye, $S$ would feel his head to be rotated toward the left and his direction of gaze toward the right. Only in this position could $S$ receive the particular pattern of stimulation provided by the lens and yet perceive the apparent frontoparallel plane veridically [ Ebenholtz, 1970, p. 344; parenthetical material not in original ]."

The difference between the visual shift hypothesis, advocated by Epstein, and the proprioceptive shift hypothesis is significant and straightforward. The first view proposes that disparity has been recalibrated, while the second view insists that the relationship between disparity and perceived depth is unaffected. Therefore, it is important to determine which is correct. The present experiment exploits the optical and perceptual properties of the overall size lens to test an implication of the contesting hypotheses.

An overall size lens (OSL) magnifies the image in all meridians. Consequently, when an OSL is worn before one eye, the binocular retinal input conforms to the image differences in the two eyes associated with asymmetrical convergence. The degree of conformity actually surpasses that produced by a MSL because there actually is no state of asymmetrical convergence that could produce an image magnification that was restricted to a single meridian. Since exposure to OSL provides a good opportunity for modification of the felt positions of head and eye, Ebenholtz's hypothesis predicts that OSL exposure should lead to an adaptive shift like that observed with the MSL.

The visual recalibration hypothesis predicts a different outcome. Basic to the prediction is the fact that an OSL, in the range of magnification studied, does not produce perceptual distortions. Perceptual distortion is absent because the vertical disparities produced by the OSL induce an opposing rotation of the frontal parallel plane that cancels the effect of the horizontal disparities. (See Ogle, 1964, Chap. 15, for a detailed discussion of the induced effect, and Williams, 1970 , for a recent demonstration of a related effect using mechanical means of introducing vertical disparity.) Since the OSL does not yield perceptual distortions, the visual system is not required to process informational discrepancy and the principal condition for adaptation in Epstein's reevaluation hypothesis is missing. Therefore, the recalibration hypothesis predicts that unlike the case of MSL exposure, OSL exposure will not yield adaptive shifts, despite the fact that OSL exposure provides an even better opportunity for proprioceptive shift than does MSL exposure.

\section{EXPERIMENT 1}

Preliminary to conduct of the main experiment, an investigation was performed to identify the stimulus conditions which would allow us to test for the presence of disparity-depth shifts while S wore an OSL before one eye. Theoretically, this may be accomplished by eliminating the basis in stimulation for the induced effect, that is, by minimizing the vertical fusion stimuli. Under such conditions, only the horizontal disparities should be effective, and the perceptual effects of an OSL should be indistinguishable from the effects of a MSL.

\section{Subjects}

The Ss were 21 undergraduates with uncorrected vision. Five of the Ss were unable to discriminate depth under the testing conditions, leaving 16 Ss as participants.

\section{Apparatus}

The depth discrimination apparatus used in the earlier experiments by Epstein and Morgan (1970) was used again. The display consists of two fine electroluminescent vertical lines, separated laterally by $65 \mathrm{~mm}$. Midway between the two lines and at the point 
Table 1

Mean Lens Effect: Objective Separation in Depth for Subjective Equidistance Effects

\begin{tabular}{lccc}
$\begin{array}{c}\text { Lens: } \\
\text { Exposure Eye }\end{array}$ & $\begin{array}{c}\text { Mean } \\
(\mathrm{cm})\end{array}$ & $\begin{array}{c}\text { Lens: } \\
\text { Exposure Eye }\end{array}$ & $\begin{array}{c}\text { Mean } \\
(\mathrm{cm})\end{array}$ \\
\hline MSL 90 Deg: L & 6.41 & MSL 90 Deg: R & 7.36 \\
MSL 180 Deg: $R$ & 0.07 & MSL 180 Deg: L & 0.07 \\
OSL: L & 6.85 & OSL: R & 7.85 \\
\hline
\end{tabular}

of objective equidistance of the lines were two fixed luminous disks, $0.8 \mathrm{~cm}$ in diam, located one above the other and separated by $1.5 \mathrm{~cm}$ between their centers. Objective equidistance was $6 \mathrm{ft}$ from $S$. The entire display was presented in total darkness. By turning a knob, $E$ caused the two lines to move slowly and continuously, one advancing and the other receding by exactly the same amount. The lines were visible to $S$ through a rectangular aperture at the face of the apparatus. The dimensions of the aperture ensured that the visible portions of both lines subtended the same invariant visual angle at all positions of the lines in the apparatus. When S's head is stationary and the room is dark, the discrimination of depth intervals between the lines depends exclusively on binocular disparity. Informal tests restricting the $S$ to monocular vision supported this claim by showing that depth discrimination was not possible without binocular vision.

\section{Lenses}

All lenses were framed in melters' goggles and worn by $S$. The lenses were: a $4 \% \mathrm{MSL}$, axis $90 \mathrm{deg}$ (vertical); a $4 \% \mathrm{MSL}$, axis $180 \mathrm{deg}$ (horizontal); a $4 \%$ OSL; and a plain glass (PG). The three pairs of goggles that had a MSL or OSL in one frame had plain glass in the other. The PG goggles had plain glass in both frames.

\section{Procedure}

The S's head was fitted into a chinrest-headclamp assembly that provided support and inhibited head movements. $\mathbf{S}$ was instructed to fixate a location between the stationary disks. Following two practice trials in setting the lines equidistant, each $S$ provided four sets of four equidistance judgments, using the method of adjustment. Eight Ss made judgments under the following conditions: MSL-90 deg, left eye; MSL-180 deg, right eye; OSL; left eye; PG. The remaining $S s$ were exposed to the lenses in front of the other eye. The four sets of judgments were made in a different order by each $\mathrm{S}$. Within each set, two of the judgments began with the left line forward and two with the right line forward. In each case, two starting positions were used.

\section{Results}

In every case, the direction of the
OSL setting conformed to the direction of the MSL-90-deg settings. For example, a $S$ wearing the MSL-90 deg before the right eye might set the right line forward to a position $7.36 \mathrm{~cm}$ in front of the left line. In this case, he would also set the right line forward by approximately the same amount while wearing the OSL in front of the right eye. The MSL-180 deg, on the other hand, proved to be ineffective; in most cases judgments made with the MSL-180 deg were indistinguishable from $P G$ judgments.

Each S's lens settings were corrected for initial response bias by expressing the lens effect as a difference between his PG setting and his lens setting, rather than as a difference between the latter and objective equidistance. Table 1 shows the mean separation in depth between the two test lines when the lines were judged to be equidistant.

\section{Conclusions}

It is obvious that under the test conditions an OSL yields apparent depth effects that are comparable in magnitude and direction to the effects produced by an MSL before the same eye. In both cases, the magnitude of the effect agreed well with the theoretical "geometric effect" of the MSL which under present conditions is $6.6 \mathrm{~cm} .1$ : Also, conforming to our understanding of the conditions of OSL effectiveness, Table 1 shows that there was no evidence of the "induced effect"; the MSL with axis horizontal, $180 \mathrm{deg}$, was ineffective. The vertical disparities produced by magnification of the separation between the disks and the lengthening of the test lines in the image of the eye wearing the lens were not sufficient to induce an effect. The absence of the induced effect is consistent with the effect obtained with the OSL and the analysis of the absence of OSL effects in unreduced environments.

\section{EXPERIMENT 2}

Experiment 2 was designed to determine if $20 \mathrm{~min}$ of OSL exposure to an extralaboratory indoor environment would lead to adaptive shifts in PG and OSL depth judgments. The results of Experiment 1 show that, under the prevailing testing conditions, depth discrimination is determined by horizontal binocular disparity. If the adaptive effects exhibited earlier by Epstein and Morgan (1970) in the case of MSL exposure were due to proprioceptive modification, the shift should also be observed in the present case. On the other hand, if MSL adaptation occurs because of the simultaneous input of discrepant visual information, then no shift is expected following OSL exposure.

\section{Subject}

Tive undergraduates were tested. Upon completion of the preadaptation stage, five Ss were discontinued because their judgments were erratic suggesting poor stereoacuity. This left $20 \mathrm{Ss}$ to serve in the full experiment.

\section{Method}

Each $S$ began by providing two practice judgments of equidistance followed by a set of four PG judgments and a set of four OSL judgments. Ten Ss were exposed to the OSL before the right eye and 10 were exposed to the OSL before the left eye. After the preadaptation series, $\mathbf{S}$ left the laboratory and walked the halls and stairs of the Psychology Building for $20 \mathrm{~min}$ accompanied by $E$. Then $\mathbf{S}$ returned to the laboratory where he provided a set of two PG judgments and a set of two OSL judgments. Half of the Ss made the PG judgments first and the others made the OSL judgments first.

\section{Results}

Table 2 shows the mean settings prior to the 20-min adaptation period. Separate analyses of variance of the PG data and the OSL data showed no significant differences among the groups. The $\mathrm{Ss}$ in Experiment 2 behaved like those in Experiment 1 by making OSL depth judgments that conformed in direction and approximate magnitude to the effects

Table 2

Mean Preadaptation (centimeters) PG and OSL Judgments

\begin{tabular}{|c|c|c|c|c|c|c|}
\hline \multirow[b]{2}{*}{$\begin{array}{c}\text { Exposure } \\
\text { Eye }\end{array}$} & \multicolumn{3}{|c|}{ PG Settings } & \multicolumn{3}{|c|}{ oSL Settings } \\
\hline & $\begin{array}{l}\text { Post PG } \\
\text { First }\end{array}$ & $\begin{array}{c}\text { Post OSL } \\
\text { First }\end{array}$ & $\begin{array}{c}\text { Both } \\
\text { Orders }\end{array}$ & $\begin{array}{c}\text { Post PG } \\
\text { First }\end{array}$ & $\begin{array}{c}\text { Post OSL } \\
\text { First }\end{array}$ & $\begin{array}{c}\text { Both } \\
\text { Orders }\end{array}$ \\
\hline Left & .65 & .61 & .63 & 8.05 & 9.68 & 8.86 \\
\hline Right & .62 & .67 & .65 & 7.38 & 8.33 & 7.84 \\
\hline Av erage & .64 & .64 & .64 & 7.72 & 9.05 & 8.35 \\
\hline
\end{tabular}


Table 3

Mean Shift (Centimeters) in Perceived Depth Following 20-Min OSL Exposure

\begin{tabular}{|c|c|c|c|c|c|c|c|c|c|}
\hline \multirow{2}{*}{$\begin{array}{c}\text { Test } \\
\text { Order }\end{array}$} & \multicolumn{3}{|c|}{ Exposed Right } & \multicolumn{3}{|c|}{ Exposed Left } & \multicolumn{3}{|c|}{ Left, Right Combined } \\
\hline & $\mathbf{N}$ & PG* & OSL + & $\mathbf{N}$ & PG & OSL & $\mathbf{N}$ & $\mathbf{P G}$ & OSL \\
\hline $\begin{array}{l}\text { PG-1 } \\
\text { OSL-1 }\end{array}$ & $\begin{array}{l}5 \\
5\end{array}$ & $\begin{array}{l}0.00 \\
0.11\end{array}$ & $\begin{array}{r}-1.64 \\
0.61\end{array}$ & $\begin{array}{l}\mathbf{5} \\
\mathbf{5}\end{array}$ & $\begin{array}{l}0.48 \\
0.28\end{array}$ & $\begin{array}{l}1.11 \\
1.74\end{array}$ & $\begin{array}{l}10 \\
10\end{array}$ & $\begin{array}{l}0.24 \\
0.20\end{array}$ & $\begin{array}{r}-0.27 \\
1.18\end{array}$ \\
\hline $\begin{array}{c}\text { Orders } \\
\text { Combined }\end{array}$ & 10 & 0.06 & -0.52 & 10 & 0.38 & 1.43 & 20 & 0.22 & 0.46 \\
\hline
\end{tabular}

*PG: Plain glass judgments assess aftereffects of adaptation.

tOSL: Overall size lens judgments are direct measures of adaptation. Negative signs designate shifts in the direction opposite to the adaptive shift.

produced by a MSL before the same eye.

Table 3 shows the mean shifts between the pre- and postexposure tests for the PG and OSL judgments. The data are fractionated into cells specified by the combinations of exposure eye and the order of judgments on the postexposure test. The PG shifts measure the aftereffect of adaptation; the OSL shifts are a direct measure of adaptation. A positive shift, i.e., an adaptive shift, is one in which the line on the same side as the exposed eye is displaced back from the frontal plane while wearing PG and toward the frontal parallel plane while wearing OSL. In other words, the objective depth interval corresponding to judged equidistance should increase for $P G$ and decrease for OSL. Separate analyses of variance of the PG and OSL shift data failed to uncover any significant effects. This establishes that neither exposure eye nor order of postexposure judgment affected the magnitude of shift. Next, the mean PG shift and mean OSL shift for all $20 \mathrm{Ss}$ combined was tested to determine if the means differed significantly from zero. Neither the PG shift $[t(19)=.993]$ nor the OSL shift $[t(19)=.892]$ differed significantly from zero.

\section{DISCUSSION}

When $\mathbf{S}$ wears an MSL before one eye, $20 \mathrm{~min}$ of exposure to an extralaboratory environment is sufficient to produce significant adaptive shifts. ${ }^{2}$ Under comparable circumstances, no adaptive shift is observed following monocular OSL exposure. For the reasons presented earlier, we believe that the difference between the effects of exposure to the two types of lenses is more compatible with the visual recalibration hypothesis of MSL adaptation than with the proprioceptive shift hypothesis. Obviously, failure to find evidence of OSL adaptation cannot be construed as support of the recalibration hypothesis. The chief significance of the finding is to place the burden of proof squarely on the advocates of the proprioceptive shift hypothesis. Positive evidence would consist of the demonstration that following MSL exposure modifications of the head-eye system are observed. A test of egocentric localization might be used for this purpose (e.g., Held \& Bossom, 1961; Hay \& Pick, 1966). On such a test, the proprioceptive shift hypothesis requires a shift of the head-eye combination that specifies a particular visual direction. At present, such evidence is lacking.

EBENHOLTZ, $S$. $M$. On the relation between interocular transfer of adaptation and Hering's law of equal innervation. Psychological Review, 1970

\section{REFERENCES} 77, 343-347.
EPSTEIN, W. Varieties of perceptual learning, New York: McGraw-Hill, 1967 EPSTEIN, W. Adaptation to uniocular image magnification after varying preadaptation activities. American Journal of Psychology, 1971, 84, 66-74. EPSTEIN, W., \& MORGAN, C. L. Adaptation to uniocular image magnification: Modification of the disparity-depth relationship. American Journal of Psychology, 1970, 73, 322-329.

HARRIS, C. S. Perceptual adaptation to inverted, reversed, and displaced vision. Psychological Review, 1965, 72, 419-444.

HAY, J. C:, \& PICK, H. L., JR. Visual and proprioceptive adaptation to prismatic displacement. Journal of Experimental Psychology, 1966, 71, 150-158.

HELD, R., \& BOSSOM, J. Neonatal deprivation and adult rearrangement: Complementary techniques for analyzing plastic sensory motor coordinations. Journal of Comparative \& Physiological Psy chology, 1961, 54, 33-37

M ACK, A., \& CHITAYAT, D. Eye-dependent and disparity adaptation to opposite visual field rotations. American Journal of Psychology, 1970 , 83, 352-371.

OGLE, K. N.Researches in binocular vision. New York: Hafner, 1964.

PICK, H., HAY, J. \& MARTIN, Adaptation to split-field wedge prism spectacles. Joumal of Experimental Psy chology, 1969, 80, 125-132.

ROCK, I. The nature of perceptual adaptation. New York: Basic Books 1966.

WILLIAMS, T. D. Vertical disparity in depth perception. American Journal of Optometry and Archives of the American Academy of Optometry, 1970, 47. 339-344.

\section{NOTES}

1. The following formula was used to compute the geometric effect: $\tan \theta=$ $[(m-1) / 2 m] X[y / a]$. where $\theta$ is the apparent rotation of the frontal plane, $m$ is lens magnification as a percentage, $y$ is observation distance, and $a$ is one-half the interocular distance. For a discussion of this equation, see Ogle (1964, pp. 160-164).

2 . In the published research, adaptation to a 5\% MSL was studied. Since the present experiment used a $4 \%$ MSL, the two conditions are not exactly comparable. However, in other work, currently in preparation for publication, adaptation to 4\% MSL was found to be significant.

(Accepted for publication May 14, 1971.) 